\title{
Balancing pre-disaster preparedness and post-disaster relief
}

\author{
Fei He*1 and Jun Zhuang ${ }^{2}$ \\ ${ }^{1}$ Department of Mechanical and Industrial Engineering, Texas A\&M \\ University-Kingsville \\ ${ }^{2}$ Department of Industrial and Systems Engineering, University at Buffalo, \\ The State University of New York
}

\begin{abstract}
Challenges associated with resource allocation to mitigate and recover from natural and man-made disasters inspire new theoretical questions for decision making in the intertwined natural and human world. Disaster loss is determined not only by postdisaster relief but also the pre-disaster mitigation and preparedness. To examine the decision making process at ex ante and ex post disaster stages, we develop a twostage dynamic programming model that optimally allocates preparedness and relief expenditures. We analytically and numerically solve the model and provide new insights by sensitivity analysis.
\end{abstract}

Keywords: Disaster, Preparedness, Relief

\section{Introduction}

Natural disasters have caused enormous damage to human beings and the economy. After Hurricane Katrina, the US government sought $\$ 105$ billion for repairs and reconstruction, and the total economic losses were about $\$ 250$ billion (King, 2005). Global natural disasters caused $\$ 350$ billion in losses in 2011 (Holm and Scism, 2011).

*Corresponding author. Tel: +1 361593 3484. Fax: +1 361593 4026. Email address: fei.he@tamuk.edu. 

identifying the characteristics and potential consequences of hazards, and insurance. Preparedness refers to measures taken to prepare for and reduce the effects of disasters. Preparedness efforts include improving the effectiveness of emergency response by developing a preparedness plan in strategic, operational, and tactical tiers, early warning systems, and public training for disaster risks and responses. Relief refers to the process of responding to a catastrophic situation, providing humanitarian aid to persons and communities who have suffered from some form of disaster. Typical efforts of relief are saving lives, protecting property and environment such as the search and rescue of human beings, and repairing and reconstructing houses. Losses caused by disasters can be reduced by not only post-disaster relief and recovery but also pre-disaster mitigation and preparedness.

The mitigation and preparedness for disasters are studied in social (Messias et al., 2012), political (Gerber, 2007) and legal (Command, 2008) contexts, such as improving the national preparedness for citizens (Conroy, 2008), integrating community/individual behaviors for disaster preparedness (Campasano, 2010), and the derivation of preparedness measurement (Covington and Simpson, 2006). Jongejan et al. (2011) conduct a cost-benefit analysis to the worthiness of preparedness. Preparedness is defined in terms of the probability of capacity exceedance to account for the response effectiveness in both densely and sparsely populated regions. Kunreuther et al. (2001), Chang (2003) and Ganderton (2005) use cost-benefit analysis to investigate the worthiness and effectiveness of mitigation via comparing the cost of mitigation and the reduction of loss and business interruption time. King (2005) and Bank and Gruber (2009) report a lack of preparedness in private sectors such as small businesses. Coffrin et al. (2011) study how to store power system supplies in the pre-disaster stage to maximize the expected power flow across all the disaster scenarios.

FEMA (2014) provides a platform to guide the public and private sectors on preparing for and recovering from disasters. There are many types of natural disasters, such as floods, tornadoes, hurricanes, thunderstorms and lightning, winter storms and extreme cold, drought, extreme heat, severe weather, space weather, earthquakes, volcanoes, landslides 
and debris flow, tsunamis, and wildfires. The mitigation and preparedness for each type of disaster could be different. For example, flooding may be caused by torrential rains, and lead to high reservoir water levels. Correspondingly, the preparedness for flooding includes the prediction of the weather, the warning system of the water level, and the reinforcement of the dam.

Disaster relief is also studied. For example, Chia (2006) analyzes disaster relief from the perspective of large-scale system engineering. Cagnan et al. (2006) study how the joint probability distribution of post-earthquake electric power restoration for a certain number of customers in a certain time window varies throughout the Los Angeles area. Altay and Green (2006) summarize the applications of operations research and management science to disaster management. Karlaftis et al. (2007) investigate the fund allocation strategy for bridge network recovery after natural disasters by maximizing bridge improvement and minimizing the cost. Stephenson and Bonabeau (2007) suggest that the government could capitalize the technology of communication devices and networks to partner with citizens to efficiently prevent and respond to disasters. Yushimito et al. (2012) propose a Voronoi diagram approach to locate the post-disaster distribution centers. The objective is to minimize the social cost which is a function of the population size and the distance from demand points to distribution centers. Haghani and Afshar (2009) propose a mathematical model to describe the integrated logistics operations in response to natural disasters.

Most research focuses on minimizing fatality or monetary loss by considering relief only, especially in a logistics perspective (Fiedrich et al., 2000, Rawls and Turnquist, 2010). There is limited research on both preparedness and relief. El-adaway and El-Anwar (2010) propose a comprehensive decision support system for natural disaster investment strategy incorporating stochastic hazards models for disaster losses, multi-agent simulation models, multi-objective optimization models and multi-attribute utility models to minimize cost and maximize equity. Kramer (1995) summarizes various risk modified cost-benefit analysis for disaster mitigation such as game-theoretic analysis, safety-first analysis, mean-variance analysis, and stochastic dominance analysis. Elbakidze and McCarl (2006) study the tradeoff of economic investment on pre-disaster preparedness and post-disaster relief for the potential introduction of infectious animal disease by minimizing the total loss of investment and 
disease damage. The disease damage is a function of preparedness, relief, and a constant incident severity parameter, while in our paper, the disaster magnitude is generally modeled as a random variable. Tean (2006) proposes a two-stage stochastic programming model to maximize the total expected number of survivors and delivery of required goods, without providing the solution of the model. Dodo et al. (2005) propose a linear model to obtain the optimal earthquake mitigation (preparedness) effort on each square footage of the region during each discrete time period, in order to minimize total costs of the mitigation and expected post-earthquake reconstruction investments. The annual probability of the occurrence of each earthquake is a constant parameter while in this paper the disaster magnitude follows a discrete or continuous distribution. Mete and Zabinsky (2010) use stochastic programming and mixed integer programming to investigate the optimal warehouse location and inventory level of the medical supply in pre-disaster stage, through solving the subproblem of minimizing the expected transportation cost after disaster. Miller-Hooks et al. (2012) set up a two-stage stochastic model to maximize the resilience of a transportation network, which is defined as the expected fraction of demand that can be satisfied for all network arcs after the disaster. In the case study, they compare the expected total post-disaster flow of shipments under the binary combinational scenarios of implementing preparedness, and/or relief. Peeta et al. (2010) build and solve a two-stage stochastic programming model to obtain the optimal pre-disaster investment for a highway network. Rose et al. (2007) shows that overall, the pre-disaster investment of one dollar has about four dollars benefit in post-disaster stage for earthquake, flood and wind hazards across US between 1993-2003. Table 1 summarizes the features of past research and presents a comparison to the research conducted in this paper. As we can see, past research mostly focuses only on either preparedness or relief. While this paper studies both preparedness and relief, and provides analytical solutions.

The damage of disaster has been categorized as direct or indirect loss; tangible or intangible loss. Tangible losses, such as physical destruction of buildings and equipment, can be evaluated by monetary values. Intangible losses are those that can not be expressed as universally accepted financial terms such as human, social, environmental and cultural losses. Several methods have been applied to estimate intangible losses such as hedonic pricing methods and travel cost methods (Department of Homeland Security, 2011; Markantonis 


\begin{tabular}{|c|c|c|c|c|}
\hline Reference & $\begin{array}{l}\text { The variable of disaster } \\
\text { magnitude }\end{array}$ & Type of hazard & $\begin{array}{l}\text { Trade-off } \\
\text { between } \\
\text { preparedness } \\
\text { and relief }\end{array}$ & $\begin{array}{l}\text { Analytical } \\
\text { Solutions } \\
\text { obtained }\end{array}$ \\
\hline $\begin{array}{l}\text { Kramer (1995); El-adaway } \\
\text { and El-Anwar (2010) }\end{array}$ & continuous and discrete & general & & \\
\hline Fiedrich et al. (2000) & continuous & earthquake & & \\
\hline Elbakidze and McCarl (2006) & constant & animal disease & $\sqrt{ }$ & \\
\hline Tean (2006) & constant & general & & \\
\hline Dodo et al. (2005) & constant & earthquake & & $\sqrt{ }$ \\
\hline $\begin{array}{l}\text { Mete and Zabinsky (2010); } \\
\text { Rawls and Turnquist (2010); } \\
\text { Miller-Hooks et al. (2012) }\end{array}$ & discrete & general & & $\sqrt{ }$ \\
\hline This research & continuous and discrete & general & $\sqrt{ }$ & $\sqrt{ }$ \\
\hline
\end{tabular}

et al., 2012). Weitzman (2011) uses multiplicative- and additive-form damage functions as a method to investigate the economic impacts from global warming. In this paper, we propose a damage function that takes into account effects of preparedness and relief besides the disaster magnitude.

The probability distribution of disaster magnitude can be described by normal distribution, exponential distribution, and power-law distribution corresponding to the trend, the extreme, and the breakdown type of disasters, respectively (Pisarenko and Rodkin, 2010). The trend, extreme, and breakdown type of disasters are categorized by the ratio of disaster magnitude to the background level from low, medium to high, respectively. Due to the scarcity of large-scale natural disaster and terrorism, it is challenging to accurately estimate the likelihood of disaster occurrence. Some exploratory methods are used to obtain the probability or distribution of catastrophes, such as Bayesian methods, catastrophe theory, entropy maximization, extreme value theory, modeling, and decomposition (Bier et al., 1999). Based on the research conducted by Starr et al. (1976), Clauset et al. (2009) and Pisarenko and Rodkin (2010), river floods, hurricane energy, droughts and moderate-term sea level variations are usually described using exponential distribution. Heights of sea waves, drought occurrence, tsunamis, tornadoes' damage swath, flood damage magnitude, and earthquake magnitude and frequency are described using lognormal distribution. The frequency of tornadoes is described using negative binomial distribution. Wind speed and wave heights are described using Rayleigh and Weibull distribution. Meteorite strikes can be described using 


\section{The Model}

The decision-making framework of prepardness and relief is shown in Figure 1. We consider two decision variables: the pre-disaster preparedness $c$; and the post-disaster relief $r(c, m)$ as a function of $c$ and $m$, where $m \geq 0$ is the disaster magnitude, which follows a discrete or continuous probability distribution $F_{M}(m)$. We consider three function forms for the disaster magnitude: (a) binary (discrete) distribution in Section 4.3; (b) normal (continuous) distribution in Section 4.4; and (c) exponential (continuous) distribution in Section 4.5. In the pre-disaster stage, the decision maker is uncertain about the disaster magnitude $m$, and decides the preparedness investment effort $c$ based on the knowledge of the probability distribution of disaster magnitude $F_{M}(m)$. In the post-disaster stage, the disaster magnitude $m$ is observed and the relief decision $r$ will be made based on $c$ and $m$. Table 2 shows all the notation used in this paper.

In the pre-disaster stage, the decision maker determines preparedness $c$ with the objective of minimizing the loss $L_{P r}$, which equals to the sum of preparedness investment, $\alpha c$ (where 
Table 2: Notation used for models of natural disaster preparedness and relief

\begin{tabular}{ll}
\hline \hline Notation & Explanation \\
\hline Parameters & Unit cost of pre-disaster preparedness effort \\
$\alpha>0$ & Unit cost of post-disaster relief effort \\
$\beta>0$ & Disaster magnitude \\
$m \geq 0$ & Thresholds for disaster magnitude \\
$h_{1}, h_{2}, h_{3}, h_{4}$ & Thresholds for pre-disaster preparedness \\
$s_{1}, s_{2}, s_{3}$ & Damage of disaster \\
$V(c, r, m)>0$ & Coefficients of the damage function \\
$V_{d}, \lambda, \xi_{1}, \xi_{2}, \xi_{3}>0$ & Probability distribution function of the disaster magnitude \\
$F_{M}(m) \geq 0$ & Probability and disaster magnitude in binary distribution \\
$P \in[0,1], m_{0}$ & Mean and Standard deviation of the disaster magnitude in normal dis- \\
$\mu_{m}>0, \sigma_{m}>0$ & tribution \\
\hline Decision variables & \\
$c \geq 0$ & Pre-disaster preparedness effort \\
$r(c, m) \geq 0$ & Post-disaster relief effort \\
$\hat{r}(c, m) \geq 0$ & Optimal post-disaster relief effort \\
\hline Losses & \\
$L_{P r}, L_{P o}>0$ & Losses in pre-disaster stage and post-disaster stage, respectively \\
\hline \hline
\end{tabular}
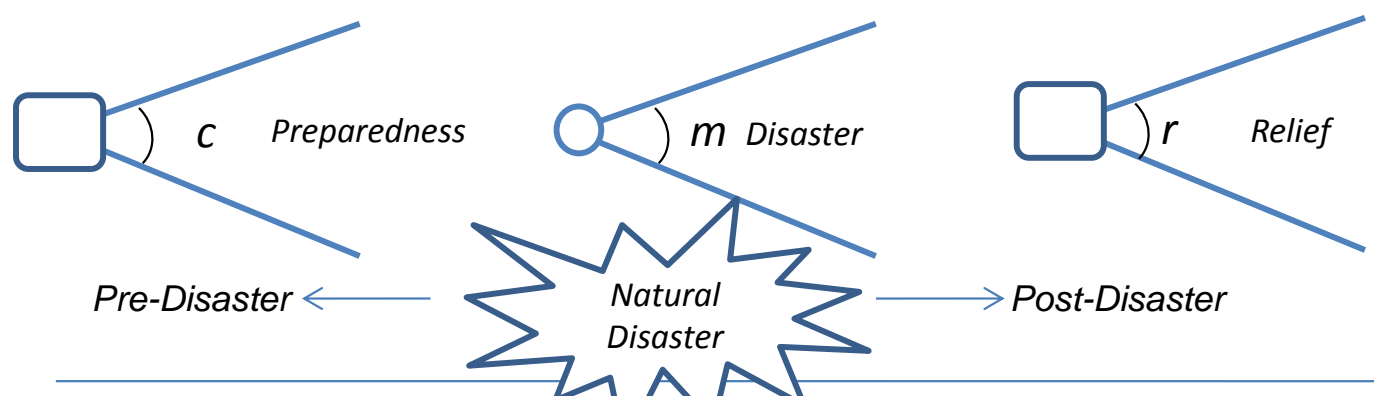

Figure 1: The sequence of decision-making on pre-disaster preparedness and post-disaster relief

$\alpha$ is the unit cost of preparedness), and the expected post-disaster loss from the disaster, $L_{P o}(c, r, m)$, as shown in Equation 1 . 


$$
\text { Pre-disaster Stage: } \min _{c \geq 0} L_{P r}=\underbrace{\alpha c}_{\begin{array}{c}
\text { pre-disaster } \\
\text { preparedness investment }
\end{array}}+\underbrace{\int_{m} L_{P o}(c, r, m) d F_{M}(m)}_{\begin{array}{c}
\text { expected post-disaster } \\
\text { loss from disaster }
\end{array}}
$$

For the post-disaster stage, the decision maker observes disaster magnitude $m$, then decides the relief effort $r$ to minimize the loss $L_{P o}$ that includes relief investment, $\beta r$ (where $\beta$ is the unit cost of relief), and damage, $V(c, r, m)$, as shown in Equation 2.

$$
\text { Post-disaster Stage: } \min _{r \geq 0} L_{P o}(c, r, m)=\underbrace{\beta r}_{\begin{array}{c}
\text { post-disaster } \\
\text { relief investment }
\end{array}}+\underbrace{V(c, r, m)}_{\begin{array}{c}
\text { damage from } \\
\text { disaster }
\end{array}}
$$

Damage functions $V(c, r, m)$ evaluate the economic damage from disaster as a function of disaster magnitude, preparedness and relief efforts. The damage of disaster $V(c, r, m)$ is assumed to be continuously differentiable, decreasing in both $c$ and $r$, and increasing in $m$, with decreasing marginal effects in $c$ and $r$. That is:

$$
\frac{\partial V}{\partial m} \geq 0, \frac{\partial V}{\partial r} \leq 0, \frac{\partial V}{\partial c} \leq 0, \frac{\partial^{2} V}{\partial c^{2}} \geq 0, \text { and } \frac{\partial^{2} V}{\partial r^{2}} \geq 0
$$

\section{The Solution}

We use backward induction to solve this two-stage dynamic model. In particular, Proposition 1 provides the solution of best-response relief to the post-disaster problem (Equation 2). Using the results in Proposition 1, Proposition 2 provides the solution to the pre-disaster problem (Equation 1).

Proposition 1. The optimal relief $\hat{r}$ of optimization problem (Equation 2), given the preparedness $c$ and the disaster magnitude $m$, is as follows.

$$
\hat{r}(c, m)= \begin{cases}\left\{r>0: \frac{\partial}{\partial r} V(c, r, m)+\beta=0\right\} & \text { if }-\left.\frac{\partial}{\partial r} V(c, r, m)\right|_{r=0}>\beta \\ 0 & \text { otherwise }\end{cases}
$$


Furthermore, we have: $\frac{\partial \hat{r}}{\partial c} \geq 0$ if and only if $\frac{\partial^{2} V}{\partial c \partial r} \leq 0 ; \frac{\partial \hat{r}}{\partial m} \geq 0$ if and only if $\frac{\partial^{2} V}{\partial m \partial r} \leq 0$.

Remark: Proposition 1 shows that the best-response relief could be positive if the marginal reduction of damage when relief is zero $\left.\frac{\partial}{\partial r} V(c, r, m)\right|_{r=0}$, is greater than the unit cost of relief $\beta$; and zero otherwise. In addition, the best-response relief $\hat{r}$ increases in the preparedness $\frac{\partial \hat{r}}{\partial c} \geq 0$, if and only if the larger marginal effect of preparedness leads to a lower level of relief $\left(\frac{\partial^{2} V}{\partial c \partial r} \leq 0\right)$. In other words, preparedness and relief are substitute to each other.

Proposition 2. The optimal preparedness $c^{*}$ of optimization problem (Equation 1), given the optimal relief $\hat{r}(c, m)$, is as follows.

$$
c^{*}=\left\{\begin{array}{l}
\left\{c>0: \frac{\partial}{\partial c} \int_{m}(\beta \hat{r}(c, m)+V(c, \hat{r}(c, m), m)) d F_{M}(m)+\alpha=0\right\} \\
\quad \text { if }-\left.\frac{\partial}{\partial c}\left\{\int_{m}(\beta \hat{r}(c, m)+V(c, \hat{r}(c, m), m)) d F_{M}(m)\right\}\right|_{c=0}>\alpha \\
\quad \text { otherwise }
\end{array}\right.
$$

Remark: Proposition 2 shows that the preparedness could be positive if the marginal reduction of the sum of relief cost and disaster damage when preparedness is zero, is less than the unit cost of preparedness; or zero otherwise. In Section 4, we explicitly derive the optimal preparedness and relief given an exponential-form damage function.

\section{Analytical Solution and Numerical Illustration for an Exponential Damage Function}

In this section, we first formulate the exponential-form damage function in Section 4.1, then derive the best-response relief based on the damage function form in Section 4.2, and analyze the optimal preparedness in Sections 4.3 when disaster magnitude follows binary, normal, and exponential distributions. 


\subsection{The Exponential Damage Function}

We assume the damage function is as follows.

$$
V(c, r, m)=\underbrace{V_{d}}_{\begin{array}{c}
\text { valuation of } \\
\text { potential loss }
\end{array}} \underbrace{\left(1-e^{-\lambda m}\right)}_{\begin{array}{c}
\text { proportion of } \\
\text { damaged targets }
\end{array}} \underbrace{e^{-\xi_{1} c-\xi_{2} r-\xi_{3} c r}}_{\begin{array}{c}
\text { discount percentage of damage } \\
\text { due to preparedness and relief }
\end{array}}
$$

The valuation of potential loss $V_{d}$ indicates both tangible and intangible loss from disaster, such as from social displacement, psychological distress, and environmental impact (Department of Homeland Security, 2011). The damage is evaluated as the valuation of potential loss $V_{d}$ reduced by the damage proportion $1-e^{-\lambda m}$, and the discount percentage of damage due to preparedness and relief effort $e^{-\xi_{1} c-\xi_{2} r-\xi_{3} c r}$. The proportion of damage is positively correlated to disaster magnitude $m$, and disaster severity parameter $\lambda$. A larger $\lambda$ leads to a higher damage $V(c, r, m)$ generated from $m$. The effectiveness of preparedness and relief are counted in three aspects: preparedness only $\xi_{1}$, relief only $\xi_{2}$, and jointly $\xi_{3}$. A larger $\xi_{1}, \xi_{2}$, or $\xi_{3}$ leads to a smaller $V$. We show that all the assumptions about the damage function presented in Equation 3 are satisfied.

Figure 2 shows the contour of the damage $V$, with respect to the preparedness $c$, and relief effort $r$. The damage increases in the disaster magnitude $m$; and decreases in $c$, and $r$. Comparing the contours on Figures 2(a) and (b) shows that the damage is about ten times larger when disaster magnitude is one hundred times larger.

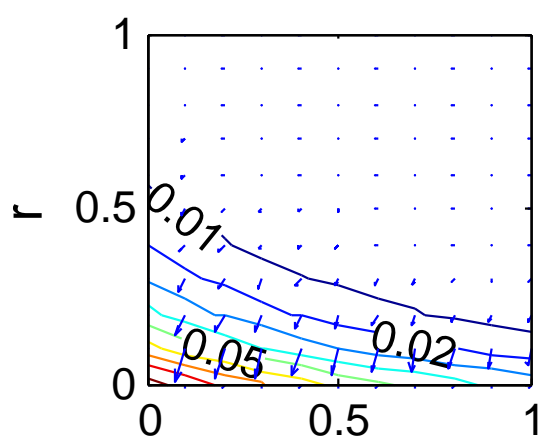

(a) C

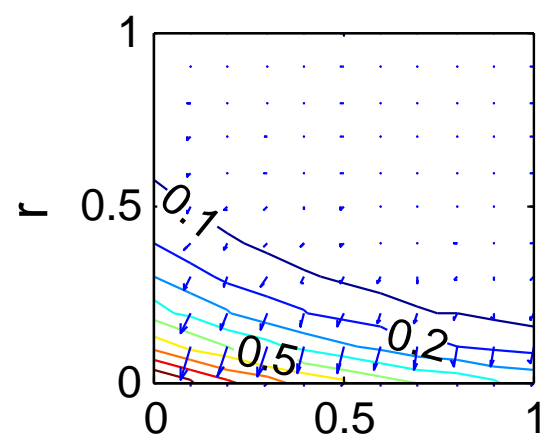

(b) C

Figure 2: Contours of the damage function $V(c, r, m)$ in the base model when (a) $\mathrm{m}=0.1$, (b) $\mathrm{m}=10$, given $V_{d}=\lambda=\xi_{1}=\xi_{2}=\xi_{3}=1$ 


\subsection{Best-Response Relief}

Using backward induction, we first solve for the best-response relief by inserting Equation 6 into Equation 4 . We use $h_{1}, \cdots, h_{4}$ to denote disaster magnitude related thresholds; and use $s_{1}, \cdots, s_{3}$ to denote preparedness related thresholds.

Proposition 3. The best-response relief $\hat{r}$, given the damage function $V(c, r, m)=V_{d}(1-$ $\left.e^{-\lambda m}\right) e^{-\xi_{1} c-\xi_{2} r-\xi_{3} c r}$, is as follows.

$$
\hat{r}(c, m)= \begin{cases}\frac{1}{\xi_{3} c+\xi_{2}}\left(\ln \frac{V_{d}\left(1-e^{-\lambda m}\right)\left(\xi_{3} c+\xi_{2}\right)}{\beta}-\xi_{1} c\right) & \text { if } s_{1}<c<s_{2}, \text { or equivalently } m>h_{1} \\ 0 & \text { otherwise }\end{cases}
$$

where the thresholds, $s_{1} \equiv-\frac{1}{\xi_{1}} W_{0}\left(\frac{\xi_{1} \beta e^{-\frac{\xi_{1} \xi_{2}}{\xi_{3}}}}{V_{d} \xi_{3}\left(e^{-\lambda m}-1\right)}\right)-\frac{\xi_{2}}{\xi_{3}}, s_{2} \equiv-\frac{1}{\xi_{1}} W_{-1}\left(\frac{\xi_{1} \beta e^{-\frac{\xi_{1} \xi_{2}}{\xi_{3}}}}{V_{d} \xi_{3}\left(e^{-\lambda m^{-}}-1\right)}\right)-\frac{\xi_{2}}{\xi_{3}}>0$, $h_{1} \equiv-\frac{1}{\lambda} \ln \left(1-\frac{\beta e^{\xi_{1} c}}{V_{d}\left(\xi_{3} c+\xi_{2}\right)}\right)$, and $W_{0}$ and $W_{-1}$ are the two real-value branches of Lambert $W$ function (Corless et al., 1996).

Remark: Proposition 3 shows that the optimal relief is positive if the preparedness satisfies $s_{1}<c<s_{2}$ (See Appendix A.3 for details). Note that the best-response relief will be zero when both the preparedness $\left(c<s_{1}\right)$ and the disaster magnitude are small, or the preparedness is too large $\left(c>s_{2}\right)$. This means that the relief is not implemented when the disaster is less likely to happen and a certain level of preparedness is sufficient to counter disasters, or when the cost versus benefit of relief is too high and it is not worthy to invest on relief. Note also that the condition $s_{1}<c<s_{2}$ in Equation 7 could be equivalently written as: $m>h_{1}$. In other words, the relief is needed if and only if the disaster magnitude is sufficiently large.

Proposition 4. The optimal relief could increase in preparedness if the preparedness and disaster magnitude are relatively small.

$$
\frac{\partial \hat{r}}{\partial c} \begin{cases}>0 & \text { if and only if } c \leq s_{3} \\ 0 & \text { otherwise }\end{cases}
$$


where the threshold $s_{3} \equiv \frac{\beta e^{1-\frac{\xi_{1} \xi_{2}}{\xi_{3}}}}{V_{d} \xi_{3}\left(1-e^{-\lambda m}\right)}-\frac{\xi_{2}}{\xi_{3}}$. When $0<s_{3}<s_{1}$, the relief $\hat{r}$, decreases in preparedness $c\left(\frac{\partial \hat{r}}{\partial c}<0\right)$; otherwise if $s_{3}>s_{1}>0, \hat{r}$ first increases in $c\left(\frac{\partial \hat{r}}{\partial c}>0\right)$ when $s_{1}<c<s_{3}$ then decreases in $c\left(\frac{\partial \hat{r}}{\partial c}<0\right)$ when $s_{3}<c<s_{2}$.

The optimal relief increases in disaster magnitude, i.e.,

$$
\frac{\partial \hat{r}}{\partial m} \geq 0
$$

Furthermore, we have

$$
\frac{\partial s_{3}}{\partial \xi_{3}}\left\{\begin{array}{ll}
\geq 0 & \text { if and only if } \xi_{1} \xi_{2} \geq \xi_{3} \\
<0 & \text { otherwise }
\end{array} \quad ; \frac{\partial s_{3}}{\partial m} \leq 0 ; \frac{\partial s_{3}}{\partial \lambda} \leq 0 ; \frac{\partial s_{3}}{\partial \xi_{1}} \leq 0 ; \text { and } \frac{\partial s_{3}}{\partial \xi_{2}} \leq 0\right.
$$

Remark: Proposition 4 implies that the relief increases in preparedness if both the disaster magnitude $m$ and the preparedness $c$ are relatively small. However, if the preparedness is large $\left(c>s_{3}\right)$ the relief decreases in preparedness. Furthermore, based on Equation 8, with a larger disaster magnitude $m$, or a lower threshold $s_{3} \geq 0$, it is more likely that post-disaster relief decreases in preparedness. With a greater effect of preparedness effectiveness, or relief effectiveness, a lower threshold $s_{3}>0$, it is more likely that post-disaster relief decreases in preparedness.

Figure 3 shows various types of best responses $\hat{r}$ with respect to $c$ when $\beta=0.5, \alpha=$ $V_{d}=\lambda=\xi_{1}=\xi_{2}=1$, and $\xi_{3}=5$. In particular, Figure 3(a) shows that the best-response relief, $\hat{r}$, is always zero for all values of preparedness, $c$, when the disaster magnitude is small $\left(m=0.5<h_{1}=0.9\right)$ and there is no need to invest in relief. Figure $3(\mathrm{~b})$ shows that $\hat{r}$ is initially zero when $c<s_{1}=1.6$, then increases monotonically between $s_{1}<c<s_{3}=4.8$ and reaches the maximum value $\hat{r}=0.82$ when $c=s_{3}$. After that, $\hat{r}$ decreases monotonically to zero between $s_{3}<c<s_{2}=24.5$ and stays at zero when $c>s_{2}$. Figure 3(c) shows that $\hat{r}$ starts as a positive value when $c=0$, then monotonically increases until $c=s_{3}=1.99$. Afterwards $\hat{r}$ starts to decrease in $c$ and remains zero when $c>s_{2}=40$. Figure 3(d) shows that $\hat{r}$ always decreases in $c$ and reaches zero when $c>s_{2}=60$. 
(a) $\mathrm{m}=0.1$

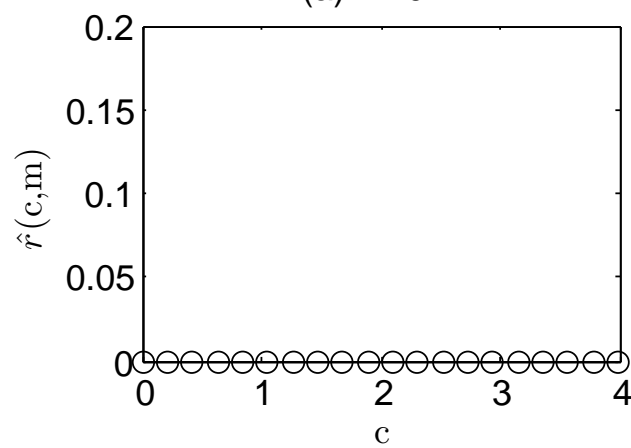

(c) $m=1$

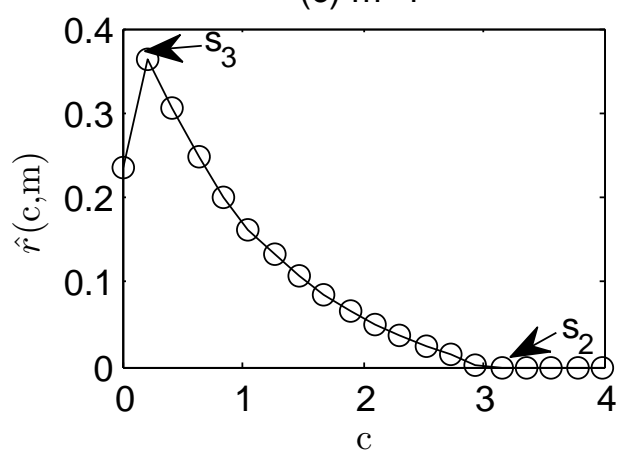

(b) $m=0.5$

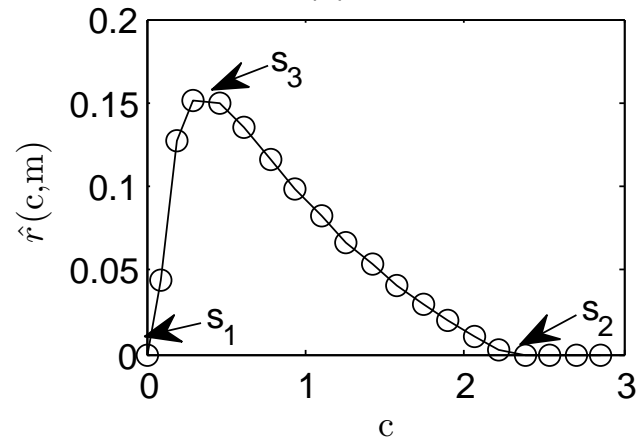

(d) $m=10$

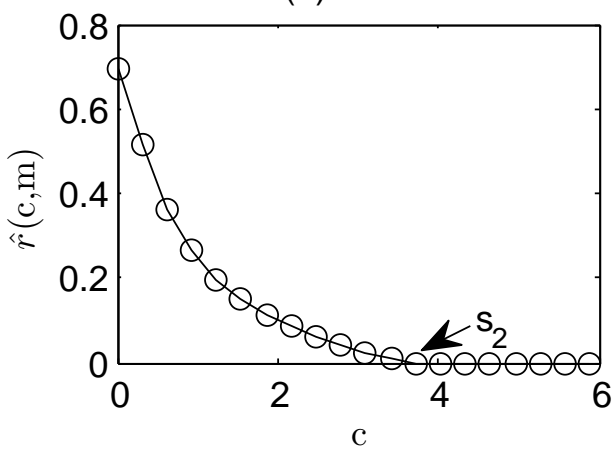

Figure 3: Optimal relief with respect to preparedness in the base model, given the parameters: $\beta=0.5, \alpha=V_{d}=\lambda=\xi_{1}=\xi_{2}=1$, and $\xi_{3}=5$

Figure 4 shows that the best-response relief increases with respect to disaster magnitude. Particularly, Figure 4(a) shows that $\hat{r}$ is zero at first, which means the preparedness is sufficient to counter the disaster. Relief $\hat{r}$ then increases to positive values when $m>h_{1}=$ 0.66 based on Equation 7, and keeps increasing in $m$. Figure 4(b) shows that positive $\hat{r}$ is always needed once the disaster happens and preparedness level is intermediate. Figure 4(c) shows that no relief is needed to counter disaster when the preparedness is large enough.
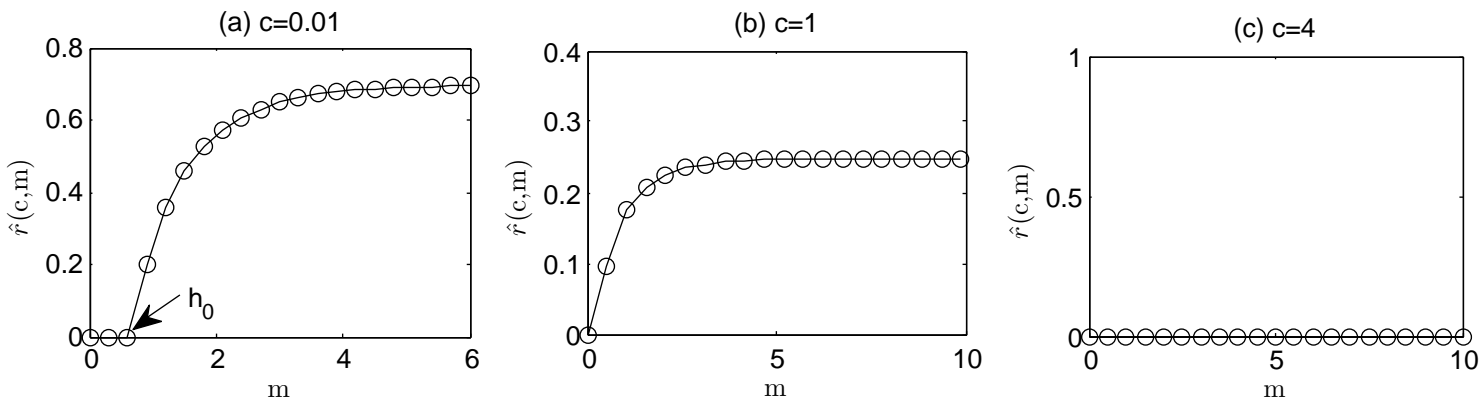

Figure 4: Optimal relief with respect to magnitude given the parameters: $\beta=V_{d}=\lambda=1$, and $\xi_{1}=\xi_{2}=\xi_{3}=0.1$ 
Figure 5 shows the optimal relief contours as a function of the preparedness and disaster magnitude. The optimal relief $\hat{r}$ first increases, then decreases in $c$ when $m$ is small; decreases in $c$ when $m$ is large; and increases in disaster magnitude $m$.

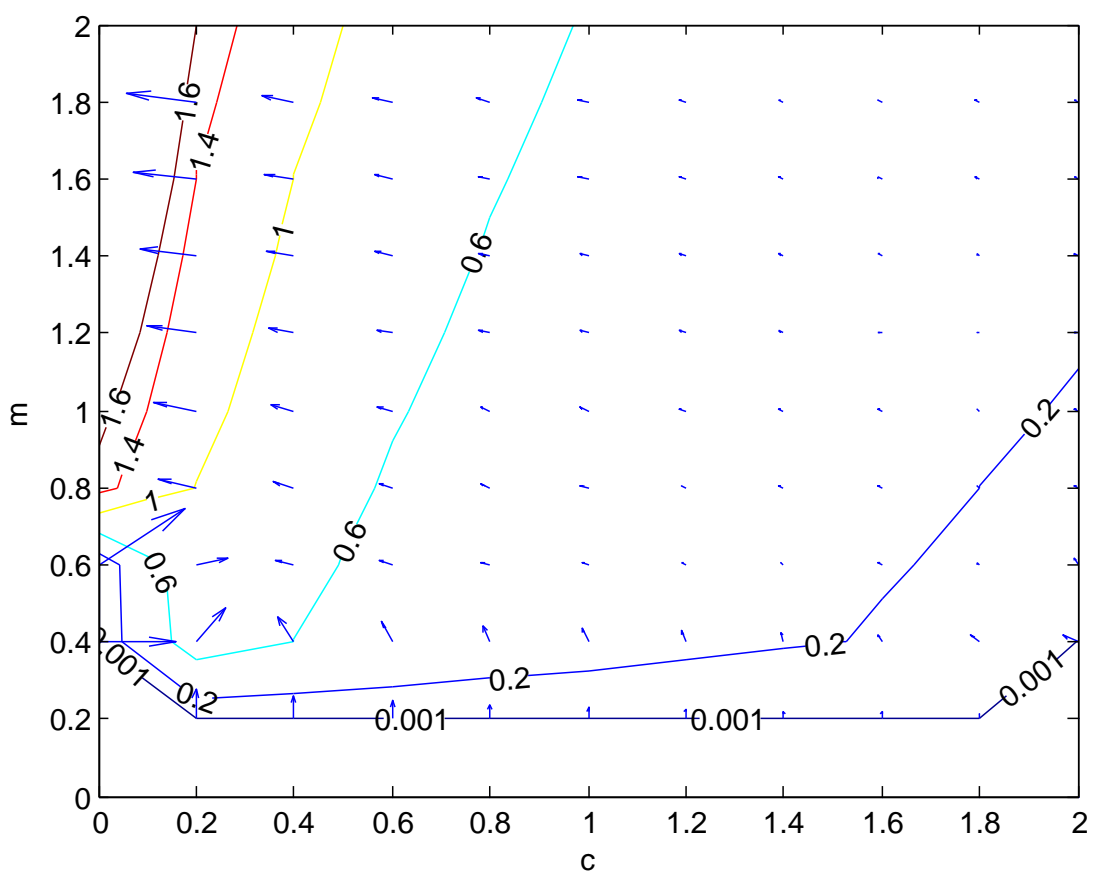

Figure 5: Optimal post-disaster relief relates to the pre-disaster preparedness and disaster magnitude in the base model, given the parameters: $\beta=\xi_{1}=V_{d}=\lambda=1, \xi_{2}=2$, and $\xi_{3}=3$

In order to explicitly solve the pre-disaster model, we need to specify the distribution of the random variable $m$. In the following sections, we show preparedness and relief when disaster magnitude follows binary, normal and exponential distributions.

\subsection{Optimal Preparedness when the Disaster Magnitude follows a Binary Distribution}

In this section, we study the simple case when magnitude $m$ follows a binary distribution.

$$
\operatorname{Pr}\left(m=m_{0}\right)=1-\operatorname{Pr}(m=0)=P
$$

Insert Equations 7 and 11 into Equation 5, we have the optimal preparedness $c^{*}$ is as follows: 


$$
c^{*}= \begin{cases}\frac{\beta e^{\lambda m}}{V_{d} \xi_{3}\left(e^{\lambda m}-1\right) e^{h_{2}}}-\frac{\xi_{2}}{\xi_{3}} & \text { if } m>h_{3} \\ 0 & \text { otherwise }\end{cases}
$$

where $h_{2} \equiv \frac{\xi_{3} \text { LambertW }\left(-\frac{2 \beta \alpha e^{\frac{2\left(\lambda m \xi_{3}-\xi_{1} \xi_{2}\right)}{\xi_{3}}}}{P V_{d}^{2}\left(e^{\lambda m}-1\right)^{2} \xi_{3}}\right)+2 \xi_{1} \xi_{2}}{2 \xi_{3}}, h_{3} \equiv-\frac{1}{\lambda \xi_{3}}\left(\xi_{1} \xi_{2}-\ln \left(\frac{\xi_{2} V_{d} e^{\frac{\xi_{1} \xi_{2}+h_{4} \xi_{3}}{\xi_{3}}}+\beta e^{\lambda}}{\xi_{2} V_{d}}\right) \xi_{3}\right.$ $\left.+h_{4} \xi_{3}\right)$, and $h_{4}$ is the root of equation $P V_{d}^{2} \xi_{3} \beta\left(e^{\lambda}\right)^{2} h_{4}+\alpha \xi_{2}^{2} V_{d}^{2}+2 \alpha \beta \xi_{2} V_{d} e^{-\frac{-\lambda \xi_{3}+\xi_{1} \xi_{2}+h_{4} \xi_{3}}{\xi_{3}}}$ $+\alpha \beta^{2} e^{-\frac{2\left(-\lambda \xi_{3}+\xi_{1} \xi_{2}+h_{4} \xi_{3}\right)}{\xi_{3}}}=0$. We notice that with the higher unit cost of relief, or the higher probability of disaster occurrence, the more likely preparedness is positive. The condition of positive preparedness shows that the preparedness is exerted when the unit cost ratio of preparedness versus relief is below a threshold, which is determined by the probability of disaster, the effectiveness of post-disaster relief, and joint effectiveness of preparedness and relief. A lower preparedness unit cost, a higher relief cost, a higher likelihood of disaster, a higher joint effectiveness, and lower relief effectiveness lead to a higher preparedness effort.

We define four cases regarding the range of preparedness and relief as the followings:

- \#1: $\left(c^{*}=0, r^{*}=0\right)$,

- \#2: $\left(c^{*}=0, r^{*}>0\right)$,

- \#3: $\left(c^{*}>0, r^{*}=0\right)$,

- \#4: $\left(c^{*}>0, r^{*}>0\right)$

Figure 6 shows the sensitivity of optimal solutions $c^{*}, r^{*}$, and $L_{P r}^{*}$ when the baseline values of the parameters are $V_{d}=1, \xi_{1}=1, \xi_{2}=4, \xi_{3}=5, \alpha=0.005, \beta=0.5, P=0.02, \lambda=$ 1 , and $m_{0}=1$. Figure $6\left(\right.$ a) shows that $c^{*}, r^{*}$, and $L_{P r}^{*}$ all increase in $m_{0}$. Relief will first increase due to the relative low disaster magnitude $\left(0<m_{0}<5.5\right)$, then the preparedness will also increase when the disaster magnitude is large $\left(m_{0}>5.5\right)$. Figure $6(\mathrm{~b})$ shows that $c^{*}$, and $L_{P r}^{*}$ increase in $P$, and $r^{*}$ decreases in $P$.

Figure 6(c) shows that as $\alpha$ increases, $c^{*}$ decreases, and $r^{*}$ and $L_{P r}^{*}$ first increase then remain at constant levels. After the preparedness decreases to zero, the relief and loss keep constant values because the unit cost of preparedness will not affect the preparedness and 


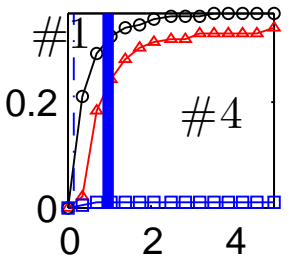

(a) $\mathrm{m}_{0}$

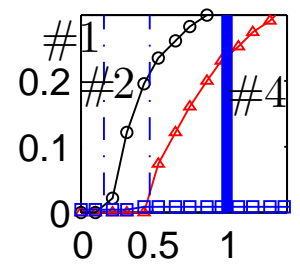

(e) $v_{0}$

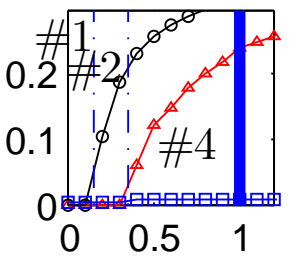

(i) $\lambda$

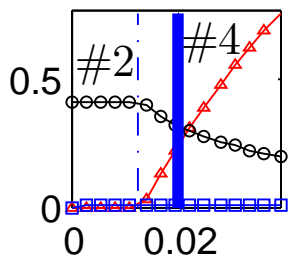

(b) $\mathrm{P}$

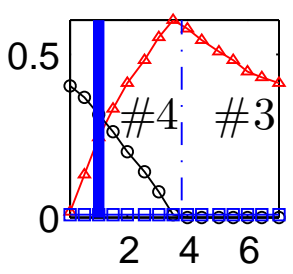

(f) $\xi_{1}$

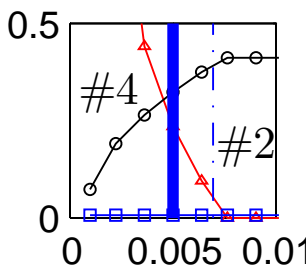

(c) $\alpha$

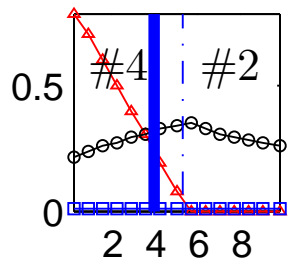

(g) $\xi_{2}$

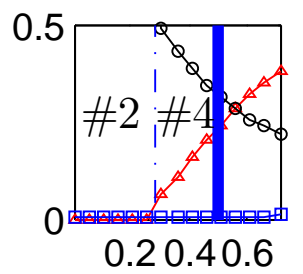

(d) $\beta$

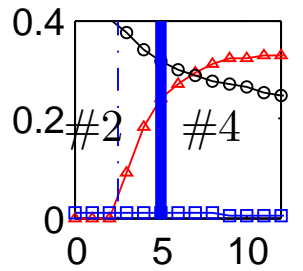

(h) $\xi_{3}$

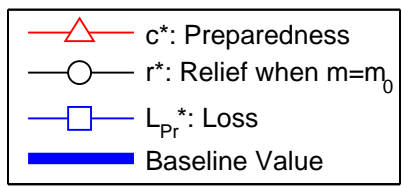

Figure 6: Sensitivity of the optimal solution when $F_{M}(m)$ follows binary distribution in the base model, given the baseline values for the parameters: $V_{d}=1, \xi_{1}=1, \xi_{2}=4, \xi_{3}=5, \alpha=$ $0.005, \beta=0.5, P=0.02, \lambda=1$, and $m_{0}=1$

relief. Figure $6(\mathrm{~d})$ shows that $r^{*}$ decreases in $\beta$. The preparedness is always zero, which means that the relief alone is efficient enough to reduce the disaster loss. The expected pre-disaster loss $L_{P r}^{*}$ first increases, then keeps at a constant level after $r^{*}$ goes to zero. Figure 6(e) shows that $c^{*}$ and $L_{P r}^{*}$ increase in the valuation of potential loss, $V_{d}$. Relief $r^{*}$ could first increase then decrease in $V_{d}$, because the relief is needed when the preparedness is relatively small and is less needed when the preparedness keeps increasing. Figure 6(f) shows that $r^{*}$ and $L_{P r}^{*}$ decrease in $\xi_{1}$, and $c^{*}$ first increases then decreases in $\xi_{1}$, which makes the equilibrium shift from case $4\left(c^{*}>0, r^{*}>0\right)$ to case $3\left(c^{*}>0, r^{*}=0\right)$. The reason is that the preparedness has little effectiveness when the preparedness effectiveness coefficient $\xi_{1}$ is small, so the optimal solution starts with a high level of relief. When $\xi_{1}$ increases the preparedness has a larger effect of loss reduction, so less relief effort is needed. As $c^{*}$ and $\xi_{1}$ increase, relief $r^{*}$ becomes unnecessary. When $\xi_{1}$ increases further, preparedness begins 
to decrease since less preparedness can effectively reduce the loss. Figure $6(\mathrm{~g})$ shows that $r^{*}$ first increases then decreases in $\xi_{2}$, preparedness $c^{*}$ is always zero, and $L_{P r}^{*}$ decreases in $\xi_{2}$. Figure $6(\mathrm{~h})$ shows that $c^{*}$ increases in $\xi_{3}$, while $r^{*}$ and $L_{P r}^{*}$ decrease in $\xi_{3}$. Due to the increasing effect of preparedness, the decision maker would invest more on preparedness to minimize the total loss and reduce the relief. Figure 6(i) shows that preparedness, relief and expected loss increase in the disaster severity parameter $\lambda$.

\subsection{Optimal Preparedness when the Disaster Magnitude follows a Normal Distribution}

When disaster magnitude $m$ follows normal distribution with mean of $\mu_{m}$ and variance of $\sigma_{m}^{2}$, we have the probability density function of left-truncated Normal distribution with non-negative values as follows.

$$
f_{M}(m)=\frac{\sqrt{2}}{\sqrt{\pi} \sigma_{m}} e^{-\frac{\left(m-\mu_{m}\right)^{2}}{2 \sigma_{m}^{2}}}, \quad \forall m>0
$$

Figure 7 shows the sensitivity of pre-disaster preparedness and the total loss for all parameters when the benefit-cost ratio between pre- and post-disaster investment $\xi_{1}: \xi_{2}$ is $4: 1$. We only consider the left-truncated normal distribution with non-negative values. Figure 7(a) shows that $c^{*}$ and $L_{P r}^{*}$ increase in $\mu_{m}$.

Figure 7(b) shows that $c^{*}$ and $L_{P r}^{*}$ first decrease then increase in $\sigma_{m}$. Specifically, when the ratio of variance versus mean of disaster magnitude is relatively small, which is less than $0.16^{2} / 1 \approx 0.026$ in this case, the optimal preparedness and the expected loss decrease in the variance. But when the ratio exceeds the threshold, 0.026, the preparedness and the expected loss increase in variance. The reason is that the probability of disaster magnitude of $\mu$ decreases when the variance starts to increase, then the preparedness decreases since the preparedness always decreases in disaster magnitude. When the variance increases to a certain high value the probability of high disaster magnitude events increases resulting in an increase in preparedness. Intuitively, the decision maker would invest solely on preparedness if there is no uncertainty of disaster magnitude. When there is a small uncertainty of disaster magnitude when variance goes to a positive value, the decision maker would allocate less 


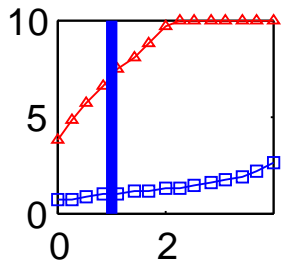

(a) $\mu_{\mathrm{m}}$

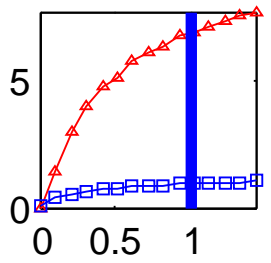

(e) $V_{d}$

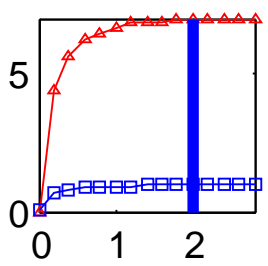

(i) $\lambda$

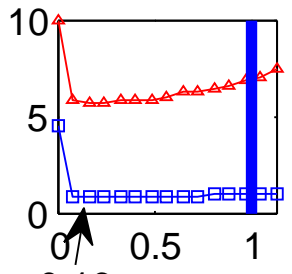

$0.16(b) \sigma_{m}$

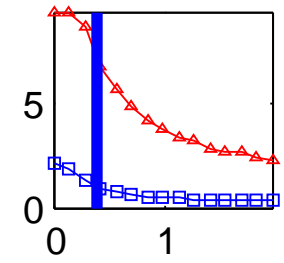

(f) $\xi_{1}$

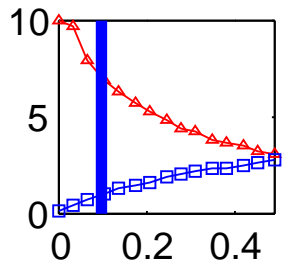

(c) $\alpha$

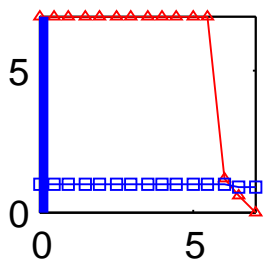

(g) $\xi_{2}$

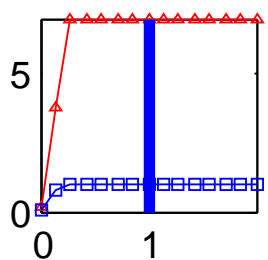

(d) $\beta$

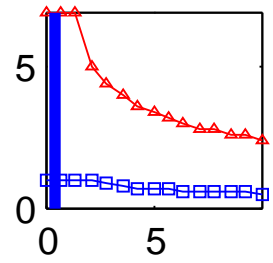

(h) $\xi_{3}$

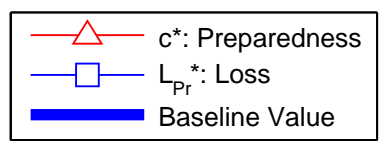

Figure 7: Sensitivity of the optimal solution when $F_{M}(m)$ follows normal distribution in the base model, given the baseline values for the parameters: $\xi_{1}=0.4, \xi_{3}=0.5, \lambda=2, \alpha=\xi_{2}=$ 0.1 , and $V_{d}=\beta=\mu_{m}=\sigma_{m}=1$

investment on preparedness and some investments for relief. When the variance increases, the decision maker would invest more on preparedness to minimize the highly uncertain disaster losses that might occur. Figure 7(c) shows that $c^{*}$ decreases in $\alpha$, and $L_{P r}^{*}$ increases in $\alpha$. Figure $7(\mathrm{~d})$ shows that both $c^{*}$ and $L_{P r}^{*}$ increase in $\beta$. This is due to the high cost of relief restraining relief investments, so more preparedness is sought. Figure 7(e) shows that both $c^{*}$ and $L_{P r}^{*}$ increase in $V_{d}$. Figure $7(\mathrm{f})$ shows that $c^{*}$ first increases then decreases in $\xi_{3}$; and $L_{P r}^{*}$ decreases in $\xi_{3}$. The reason is that less preparedness is needed when the joint preparedness and relief is more effective. Figure $7(\mathrm{~g})$ shows that $c^{*}$ first increases then decreases in $\xi_{3}$. The reason is that less preparedness is needed if preparedness is more effective. The loss $L_{P r}^{*}$ decreases in $\xi_{3}$. Figure $7(\mathrm{~h})$ shows that $c^{*}$ and $L_{P r}^{*}$ decrease in $\xi_{2}$. As expected the greater effectiveness of relief, the less preparedness is needed. 


\subsection{Optimal Preparedness when the Disaster Magnitude follows an Exponential Distribution}

Based on the fact that the probability of disaster occurrence exponentially decreases with the increase of disaster magnitude (Starr et al., 1976, Bier et al., 2008), we assume that the probability density of disaster magnitude has the following form

$$
f_{M}(m)=a e^{-a m}, \quad \forall m \geq 0
$$

where $a>0$. Notice that $a$ is the probability density of disaster magnitude when the magnitude equals to zero. We find similar effects as shown in Sections 4.3 and 4.4. Specifically, preparedness decreases and expected loss increases in unit cost of preparedness; preparedness increases in unit cost of relief; preparedness and expected loss increase in target valuation; preparedness and expected loss decrease in preparedness effectiveness; and preparedness increases in disaster severity. Figure $8(\mathrm{a})$ shows that when $F_{M}(m)$ follows the exponential distribution (Equation 13), preparedness first increases then decreases in the coefficient $a$, which negatively relates to the likelihood of disaster occurrence. The expected loss also decreases.
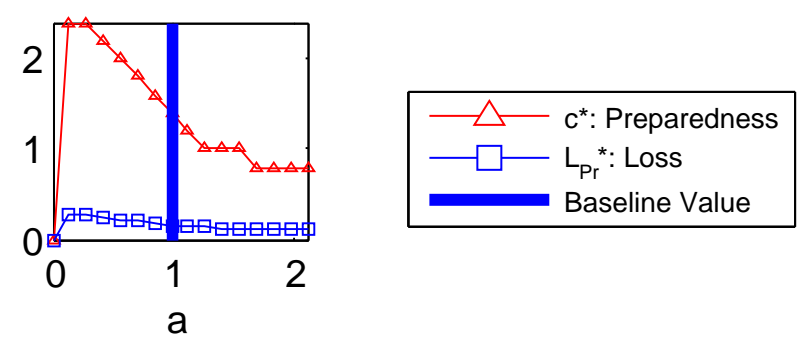

Figure 8: Sensitivity of the optimal solution when $F_{M}(m)$ follows the exponential distribution, given the baseline values for the parameters: $a=-1, \xi_{1}=4, \xi_{3}=5, \alpha=0.1$, and $\beta=\xi_{2}=V_{d}=\lambda=1$

\section{Conclusion and Future Research Directions}

In order to balance pre-disaster preparedness and post-disaster relief, we develop a twostage dynamic model to minimize the total loss in both pre- and post-disaster stages. We use 
backward induction to obtain optimal preparedness and relief both analytically and numerically. Our model reveals insights for seeking the optimal policy on balancing pre-disaster preparedness and post-disaster relief for local, state, federal government or business units that are facing potential disasters. Our results show that relief can increase in preparedness (especially when disaster magnitude and preparedness are small), and eventually decrease in preparedness. The relief always increases in disaster magnitude. The preparedness decreases in the unit cost of preparedness but increases in the unit cost of relief. The preparedness and total loss first decrease then increase in the variance of the disaster magnitude.

In the future, the effects of other important factors during disaster management can be analyzed such as budget constraint, interdependence between multiple affected locations, allhazard including adaptive disasters such as terrorism, and multiple-period decision-making of mitigation and relief. We are also interested in utilizing data analytics on real disaster investment and historical damage data (e.g., Centre for Research on the Epidemiology of Disasters, 2015) to validate model solutions.

\section{Acknowledgements}

The authors would like to thank the financial supports from the National Center for Risk and Economic Analysis of Terrorism Events of United States Department of Homeland Security under award number 2010-ST-061-RE0001, and U.S. National Science Foundation through Award No. 1334930. However, any opinions, findings, and conclusions or recommendations in this document are those of the authors and do not necessarily reflect views of the sponsors. 


\section{A Proof of Propositions}

\section{A.1 Proof of Proposition 1}

Proof. Since Equation 2 has a minimum solution at $\hat{r}$, and $V(c, r, m)$ is differentiable at $\hat{r}$, then

$$
\frac{\partial L_{P_{o}}}{\partial r}=\frac{\partial}{\partial r}(\beta r+V(c, r, m))=\frac{\partial}{\partial r} V(c, r, m)+\beta=0
$$

There are two possibilities according to the value of $\left.\frac{\partial L_{P o}(c, r, m)}{\partial r}\right|_{r=0}$ :

1. $\left.\frac{\partial L_{P o}(c, r, m)}{\partial r}\right|_{r=0}>0$. Since $V(c, r, m)$ is convex in $r$ (see Equation 3), then we have $\left.\frac{\partial V(c, r, m)}{\partial r}\right|_{r>0} \geq\left.\frac{\partial V(c, r, m)}{\partial r}\right|_{r=0}$, so we have $\left.\frac{\partial L_{P o}(c, r, m)}{\partial r}\right|_{r>0} \geq\left.\frac{\partial L_{P o}(c, r, m)}{\partial r}\right|_{r=0}>0$. Equation A.1 will not hold for any $\hat{r}(c, m)>0$. Therefore, the solution is $\hat{r}(c, m)=0$.

2. $\left.\frac{\partial L_{P o}(c, r, m)}{\partial r}\right|_{r=0} \leq 0$. Since $V(c, r, m)$ is convex in $r$, then we have $\left.\frac{\partial V(c, r, m)}{\partial r}\right|_{r>0} \geq$ $\left.\frac{\partial V(c, r, m)}{\partial r}\right|_{r=0}$. There must exist an $r>0$ such that $\left.\frac{\partial L_{P o}(c, m, r)}{\partial r}\right|_{r>0} \geq\left.\frac{\partial L_{P o}(c, m, r)}{\partial r}\right|_{r=0}=0$. Therefore, the solution is $\left\{r: \frac{\partial V(c, r, m)}{\partial r}+\beta=0\right\}$

In addition, the second-order condition for a minimum solution is checked as the following: $\frac{\partial^{2} L_{P o}}{\partial r^{2}}=\frac{\partial^{2}}{\partial r^{2}} V(c, r, m) \geq 0$ because the damage function $V(c, r, m)$ is convex in $r$. So, we have Equation 4.

Furthermore, since $\hat{r}(c, m)=0$ when $\left.\frac{\partial}{\partial r} V(c, r, m)\right|_{r=0} \geq-\beta$. We only need consider the case of $\hat{r}(c, m)>0$. Taking the derivative of Equation 4 with respect to $c$, we have $\frac{\partial^{2} V}{\partial c \partial r}+\frac{\partial^{2} V}{\partial r^{2}} \frac{\partial r}{\partial c}=$ 0 , which is equivalent to $\frac{\partial r}{\partial c}=-\frac{\partial^{2} V}{\partial c \partial r} / \frac{\partial^{2} V}{\partial r^{2}}$. Because $\frac{\partial^{2} V}{\partial r^{2}} \geq 0$, we have $\frac{\partial \hat{r}}{\partial c} \geq 0$ if $\frac{\partial^{2} V}{\partial c \partial r} \leq 0$. Similarly, taking the derivative of Equation 4 with respect to $m$, we have $\frac{\partial \hat{r}}{\partial m} \geq 0$ if $\frac{\partial^{2} V}{\partial m \partial r} \leq 0$.

\section{A.2 Proof of Proposition 2}

In order to prove Proposition 2, we show both necessary and sufficient conditions for $c^{*}$ to be a solution as specialized in Equation 5.

Proof. The existence of maximum solution of Equation 1 requires that

$$
\frac{\partial L_{P r}}{\partial c}=\frac{\partial}{\partial c} \int_{m}(\beta r+V) d F_{M}(m)+\alpha=0
$$

When $\hat{r}(c)$ is convex in $c$, there are two possibilities: 
1. $\left.\frac{\partial L_{P_{r}}(c, r, m)}{\partial c}\right|_{c=0}>0$. Since both $V(c, r, m)$ and $\hat{r}$ are convex in $c$, we have $\left.\frac{\partial}{\partial c} \int_{m}(\beta r+V) d F_{M}(m)\right|_{c>0}>\left.\frac{\partial}{\partial c} \int_{m}(\beta r+V) d F_{M}(m)\right|_{c=0}$, based on the fact that the convexity is preserved under nonnegative scaling and addition (Boyd and Vandenberghe, 2004). Therefore Equation A.2 will not hold for any $c^{*}>0$. Therefore, the solution is $c^{*}=0$.

2. $\left.\frac{\partial L_{P r}(c, r, m)}{\partial c}\right|_{c=0} \leq 0$. Since both $V(c, r, m)$ and $\hat{r}$ are convex in $c$, there must exist $c>0$ such that $\frac{\partial \bar{L}_{P r}}{\partial c}=0$. Therefore the solution is $\left\{c: \frac{\partial}{\partial c} \int_{m}(\beta r+V) d F_{M}(m)+\alpha=0\right\}$

In addition, based on Leibniz integral rule (Bartle and Sherbert, 2007), when $\beta \hat{r}(c, m)+$ $V(c, \hat{r}(c, m), m)$ and $\frac{\partial}{\partial c}(\beta \hat{r}(c, m)+V(c, \hat{r}(c, m), m))$ are both continuous in $c$ and $m$, the derivative can be taken inside the integral. So we have

$$
c^{*}=\left\{\begin{array}{l}
\left\{c: \int_{m} \frac{\partial}{\partial c}(\beta \hat{r}(c, m)+V(c, \hat{r}(c, m), m)) d F_{M}(m)+\alpha=0\right\} \\
0 \quad \text { if }-\left.\int_{m} \frac{\partial}{\partial c}(\beta \hat{r}(c, m)+V(c, \hat{r}(c, m), m)) d F_{M}(m)\right|_{c=0} \geq \alpha \\
\text { otherwise }
\end{array}\right.
$$

In the following, we show the solution in Equation 5 is sufficiently optimal.

$$
\begin{aligned}
\frac{\partial^{2} L_{P r}}{\partial c^{2}} & =\frac{\partial}{\partial c}\left(\frac{\partial}{\partial c}\left(\int_{m}(\beta r(c, m)+V(c, r(c, m), m)) d F_{M}(m)\right)+\alpha\right) \\
& =\int_{m}\left(\frac{\partial^{2} V(c, r, m)}{\partial c^{2}}+\beta \frac{\partial^{2} \hat{r}(c, m)}{\partial c^{2}}\right) d F_{M}(m)
\end{aligned}
$$

We assume $-\frac{1}{\beta} \frac{\partial^{2} V(c, r, m)}{\partial c^{2}} \leq \frac{\partial^{2} \hat{r}(c, m)}{\partial c^{2}} \leq 0$, such that $\frac{\partial^{2} V(c, r, m)}{\partial c^{2}}+\beta \frac{\partial^{2} \hat{r}(c, m)}{\partial c^{2}} \geq 0$. So we have $L_{P r}(c, r, m)$ is positive semidefinite. Therefore, $c^{*}$ in Equation 5 is a global optimal solution.

\section{A.3 Proof of Proposition 3}

Based on Equation 4, if $\left.\frac{\partial}{\partial r} V(c, r, m)\right|_{r=0} \leq-\beta$, then we have $\hat{r}=\left\{r: \frac{\partial}{\partial r} V(c, r, m)+\beta=\right.$ $0\}$. Plugged into the function form of $V(c, r, m)=V_{d}\left(1-e^{-\lambda m}\right) e^{-\xi_{1} c-\xi_{2} r-\xi_{3} c r}, \hat{r}$ becomes the following:

$$
\hat{r}=\frac{1}{\xi_{3} c+\xi_{2}}\left(\ln \frac{V_{d}\left(1-e^{-\lambda m}\right)\left(\xi_{3} c+\xi_{2}\right)}{\beta}-\xi_{1} c\right)
$$

Meanwhile, the condition $\left.\frac{\partial}{\partial r} V(c, r, m)\right|_{r=0} \leq-\beta$ turns out to be

$$
m \geq-\frac{1}{\lambda} \ln \left(1-\frac{\beta e^{\xi_{1} c}}{V_{d}\left(\xi_{3} c+\xi_{2}\right)}\right)
$$


which can be simplified in terms of $c$ :

$$
s_{1} \leq c \leq s_{2}
$$

where $s_{1}=-\frac{1}{\xi_{1}} W_{0}\left(\frac{\xi_{1} \beta e^{-\frac{\xi_{1} \xi_{2}}{\xi_{3}}}}{V_{d} \xi_{3}\left(e^{-\lambda m}-1\right)}\right)-\frac{\xi_{2}}{\xi_{3}}, s_{2}=-\frac{1}{\xi_{1}} W_{-1}\left(\frac{\xi_{1} \beta e^{-\frac{\xi_{1} \xi_{2}}{\xi_{3}}}}{V_{d} \xi_{3}\left(e^{-\lambda m}-1\right)}\right)-\frac{\xi_{2}}{\xi_{3}}$. Denoting the right hand side of Equation A.5 as $h_{1}$, the solution for Equation A.4 is:

$$
\hat{r}(c, m)= \begin{cases}\frac{1}{\xi_{3} c+\xi_{2}}\left(\ln \frac{V_{d}\left(1-e^{-\lambda m}\right)\left(\xi_{3} c+\xi_{2}\right)}{\beta}-\xi_{1} c\right) & \text { if } s_{1} \leq c \leq s_{2} \text { or } m \geq h_{1} \\ 0 & \text { otherwise }\end{cases}
$$

\section{A.4 Proof of Proposition 4}

Let $\frac{\partial \hat{r}}{\partial c} \geq 0 \Rightarrow \frac{\partial}{\partial c}\left(\frac{1}{\xi_{3} c+\xi_{2}}\left(\ln \frac{V_{d}\left(1-e^{-\lambda m}\right)\left(\xi_{3} c+\xi_{2}\right)}{\beta}-\xi_{1} c\right)\right) \geq 0$, solving for $c$ to get

$$
c \leq s_{3}
$$

where $s_{3}=\frac{1}{\xi_{3}}\left(\frac{\beta e^{1-\frac{\xi_{1} \xi_{2}}{\xi_{3}}}}{V_{d}\left(1-e^{-\lambda m}\right)}-\xi_{2}\right)$. So we have $\frac{\partial \hat{r}}{\partial c} \geq 0$ if and only if $c \leq s_{3}$.

From the feasibility requirement of Lambert $W$ function in Equation A.6, $-\frac{1}{e} \frac{\xi_{1} \beta e^{-\frac{\xi_{1} \xi_{2}}{\xi_{3}}}}{V_{d} \xi_{3}\left(e^{-\lambda m}-1\right)} \leq$ $0 \Rightarrow \frac{\beta e^{1-\frac{\xi_{1} \xi_{2}}{\xi_{3}}}}{V_{d}\left(1-e^{-\lambda m}\right)} \leq \frac{\xi_{3}}{\xi_{1}}$, then $s_{3} \leq-\frac{\xi_{2}}{\xi_{3}}+\frac{1}{\xi_{1}}$. From the above $s_{2} \geq-\frac{\xi_{2}}{\xi_{3}}+\frac{1}{\xi_{1}}$, So $s_{3} \leq s_{2}$. Based on the comparison of $s_{3}$ and $s_{1}$ we find the monotonic range of the relief changing with respect to the preparedness on the following conditions:

- When $s_{3} \leq s_{1}$, the relief decreases in preparedness when $s_{1} \leq c \leq s_{2}$;

- When $s_{3}>s_{1}$, the relief first increases in preparedness when $s_{1} \leq c<s_{3}$, then decreases when $s_{3} \leq c \leq s_{2}$ in preparedness.

In the following calculations, we prove Equation 10. Based on Equation 8,

$\frac{\partial s_{3}}{\partial m}=\frac{\partial}{\partial m}\left(\frac{1}{\xi_{3}}\left(\frac{\beta e^{1-\frac{\xi_{1} \xi_{2}}{\xi_{3}}}}{V_{d}\left(1-e^{-\lambda m}\right)}-\xi_{2}\right)\right)=-\frac{\beta \lambda e^{-\lambda m-\frac{\xi_{1} \xi_{2}}{\xi_{3}}+1}}{V_{d} \xi_{3}\left(-1+e^{-\lambda m}\right)^{2}} \leq 0$.

$\frac{\partial s_{3}}{\partial \xi_{3}}=\frac{\partial}{\partial \xi_{3}}\left[\frac{1}{\xi_{3}}\left(\frac{\beta e^{1-\frac{\xi_{1} \xi_{2}}{\xi_{3}}}}{V_{d}\left(1-e^{-\lambda m}\right)}-\xi_{2}\right)\right]=\frac{\xi_{2}}{\xi_{3}^{2}}+\frac{\left(\xi_{1} \xi_{2}-\xi_{3}\right) \beta e^{1-\frac{\xi_{1} \xi_{2}}{\xi_{3}}}}{\xi_{2}^{3} V_{d}\left(1-e^{-\lambda m}\right)}$, If $\xi_{1} \xi_{2}>\xi_{3}$, then $\frac{\partial s_{3}}{\partial \xi_{3}}>0$; else if $\xi_{1} \xi_{2} \leq \xi_{3}, \frac{\partial s_{3}}{\partial \xi_{3}} \leq 0$

$$
\begin{gathered}
\frac{\partial s_{3}}{\partial \xi_{1}}=\frac{\partial}{\partial \xi_{1}}\left[\frac{1}{\xi_{3}}\left(\frac{\beta e^{1-\frac{\xi_{1} \xi_{2}}{\xi_{3}}}}{V_{d}\left(1-e^{-\lambda m}\right)}-\xi_{2}\right)\right]=\frac{\xi_{2} \beta e^{1-\frac{\xi_{1} \xi_{2}}{\xi_{3}}}}{\xi_{3}^{2} V_{d}\left(-1+e^{-\lambda m}\right)} \leq 0 . \\
\frac{\partial s_{3}}{\partial \xi_{2}}=\frac{\partial}{\partial \xi_{2}}\left[\frac{1}{\xi_{3}}\left(\frac{\beta e^{1-\frac{\xi_{1} \xi_{2}}{\xi_{3}}}}{V_{d}\left(1-e^{-\lambda m}\right)}-\xi_{2}\right)\right]=-\frac{1}{\xi_{3}}+\frac{\xi_{1} \beta e^{1-\frac{\xi_{1} \xi_{2}}{\xi_{3}}}}{\xi_{3}^{2} V_{d}\left(-1+e^{-\lambda m}\right)} \leq 0 \\
\frac{\partial s_{3}}{\partial \lambda}=\frac{\partial}{\partial \xi_{2}}\left[\frac{1}{\xi_{3}}\left(\frac{\beta e^{1-\frac{\xi_{1} \xi_{2}}{\xi_{3}}}}{V_{d}\left(1-e^{-\lambda m}\right)}-\xi_{2}\right)\right]=-\frac{\beta m e^{1-\lambda m-\frac{\xi_{1} \xi_{2}}{\xi_{3}}}}{\xi_{3} V_{d}\left(-1+e^{-\lambda m}\right)^{2}} \leq 0 . \text { So we have Equation } 10 .
\end{gathered}
$$


Regarding the best relief with respect to disaster magnitude, when $\hat{r}>0$, we take the derivative of $\hat{r}$ with respect to $m$ in Equation 7:

$$
\frac{\partial \hat{r}}{\partial m}=\frac{\partial}{\partial m}\left(\frac{1}{\xi_{3} c+\xi_{2}}\left(\ln \frac{V_{d}\left(1-e^{-\lambda m}\right)\left(\xi_{3} c+\xi_{2}\right)}{\beta}-\xi_{1} c\right)\right)=\frac{\lambda e^{-\lambda m}}{\left(1-e^{-\lambda m}\right)\left(\xi_{3} c+\xi_{2}\right)} \geq 0
$$

where $\lambda \geq 0$. So we have Equation 9 . 


\section{References}

Altay, N., W. G. Green. 2006. Or/ms research in disaster operations management. European Journal of Operational Research 175(1) 475-493.

Bank, M., M. Gruber. 2009. Behaviouristic approaches to insurance decisions in the context of natural hazards. Safety, Reliability and Risk Analysis: Theory, Methods and Applications. Taylor and Francis Group, London, UK, 2675-2683.

Bartle, R. G., D. R. Sherbert. 2007. Introduction to Real Analysis. 3rd ed. Wiley, Hoboken, NJ.

Bier, V. M., Y. Y. Haimes, J. H. Lambert, N. C. Matalas, R. Zimmerman. 1999. A survey of approaches for assessing and managing the risk of extremes. Risk analysis 19(1) 83-94.

Bier, V. M., N. Haphuriwat, J. Menoyo, R. Zimmerman, A. M. Culpen. 2008. Optimal resource allocation for defense of targets based on differing measures of attractiveness. Risk Analysis 28(3) $763-770$.

Boyd, S., L. Vandenberghe. 2004. Convex Optimization. Cambridge University Press, Cambridge, United Kingdom.

Cagnan, Z., R. A. Davidson, S. D. Guikema. 2006. Post-earthquake restoration planning for los angeles electric poweer. Earthquake Spectra 22(3) 589-608.

Campasano, N. 2010. Community preparedness: creating a model for change. Master's thesis, Naval Postgraduate School, Monterey, CA.

Centre for Research on the Epidemiology of Disasters. 2015. EM-DAT online database Universite' Catholique de Louvain in Brussels, Belgium. http://www.emdat.be. Accessed November 2015.

Chang, S. E. 2003. Evaluating disaster mitigations; methodology for urban infrastructure systems. Natural Hazards Review 4(4) 186-196.

Chia, E. S. 2006. Engineering disaster relief. International Symposium on Technology and Society, Proceedings, 2006. 1-5.

Clauset, A., C. R. Shalizi, M. E. J. Newman. 2009. Power-law distributions in empirical data. SIAM review 51(4) 661-703.

Coffrin, C., P. V. Hentenryck, R. Bent. 2011. Strategic stockpiling of power system supplies for disaster recovery. IEEE PES 2011. IEEE Power \& Energy Society, Detroit, MI.

Command, United States Joint Forces. 2008. Legal aspects: Civilians/contractors in the war zone and disaster relief support. Joint Center for Operational Analysis Journal 10(2) 1-64.

Conroy, A. 2008. What is going to move the needle on citizen preparedness? can america create culture of preparedness. Master's thesis, Naval Postgraduate School, Monterey, CA.

Corless, R. M., G. H. Gonnet, D. E. G. Hare, D. J. Jeffrey, D. E. Knuth. 1996. On the lambert w function. Advances in Computational Mathematics 5(4) 329-359.

Covington, J., D. M. Simpson. 2006. An overview of disaster preparedness literature: Building blocks for an applied bay area template. http://hazardcenter.louisville.edu/pdfs/wp0602.pdf. Accessed December 2014.

Department of Homeland Security. 2011. The strategic national risk assessment in support of ppd 8: A comprehensie risk-based approach toward a secure and resilient nation. http://www.dhs.gov/xlibrary/assets/rma-strategic-national-risk-assessment-ppd8.pdf. Accessed December 2014.

Dodo, A., N. Xu, R. A. Davidson, L. K. Nozick. 2005. Optimizing regional earthquake mitigation investment strategies. Earthquake Spectra 21(2) 305-327. 
El-adaway, I. H., O. H. El-Anwar. 2010. Developing optimal hazard investment strategies. http://ascelibrary.org/doi/pdf/10.1061/41109 Accessed December 2014.

Elbakidze, L., B. McCarl. 2006. Animal disease pre event preparedness versus post event response: When is it economic to protect? Journal of Agricultural and Applied Economics 38(2) 327-336.

FEMA. 2014. Natural disasters. http://www.ready.gov. Accessed August 2014.

Fiedrich, F., F. Gehbauer, U. Rickers. 2000. Optimized resource allocation for emergency response after earthquake disasters. Safety Science 35(1) 41-57.

Ganderton, P. T. 2005. 'benefit-cost analysis' of disaster mitigation: Application as a policy and decision-making tool. Mitigation and Adaptation Strategies for Global Change 10(3) 445-465.

Gerber, B. J. 2007. Disaster management in the united states: Examining key political and policy challenges. Policy Studies Journal 35(2) 227-238.

Haghani, A., A. Afshar. 2009. Supply chain management in disaster response. http://www.citsm.umd.edu/documents/abstracts-finished/haghani2-abstract.php. Accessed December 2014.

Holm, E., L. Scism. 2011. Disaster losses hit record levels in 2011. http://online.wsj.com/article/SB10001424052970204026804577099820029507292.html. Accessed December 2014.

Jongejan, R. B., I. Helsloot, R. J. Beerens, J. K. Vrijling. 2011. How prepared is prepared enough? Disasters 35(1) 130-142.

Karlaftis, M. G., K. L. Kepaptsoglou, S. Lambropoulos. 2007. Fund allocation for transportation network recovery following natural disasters. Journal of Urban Planning and Development 133(1) 82-89.

King, R. O. 2005. Hurricane katrina: Insurance losses and national capacities for financing disaster risk. http://www.gpo.gov/fdsys/pkg/CRPT-109srpt322/pdf/CRPT-109srpt322.pdf. Accessed December 2014.

Kramer, R. A. 1995. Advantages and limitations of benefit-cost analysis for evaluating investments in natural disaster mitigation. http://desastres.unanleon.edu.ni/pdf/2002/septiembre/pdf/eng/doc6540/doc6540-a.pdf. Accessed December 2014.

Kunreuther, H., P. Grossi C. Cyr, W. Tao. 2001. Using cost-benefit analysis to evaluate mitigation for lifeline systems. http://grace.wharton.upenn.edu/risk/downloads/01-14-HK.pdf. Accessed December 2014.

Lindsay, Bruce R. 2012. Federal emergency management: A brief introduction. Congressional Research Service, Library of Congress.

Markantonis, V., V. Meyer, R. Schwarze. 2012. Valuating the intangible effects of natural hazards-review and analysis of the costing methods. http://www.nat-hazards-earth-systsci.net/12/1633/2012/nhess-12-1633-2012.pdf.

Messias, D. K. H., C. Barrington, E. Lacy. 2012. Latino social network dynamics and the hurricane katrina disaster. Disasters 36(1) 101-121.

Mete, H. O., Z. B. Zabinsky. 2010. Stochastic optimization of medical supply location and distribution in disaster management. International Journal of Production Economics 126 76-84.

Miller-Hooks, E., X. Zhang, R. Faturechi. 2012. Measuring and maximizing resilience of freight transportation networks. Computers and Operations Research 39(7) 1633-1643.

Peeta, S., F. S. Salman, D. Gunnec, K. Viswanath. 2010. Pre-disaster investment decisions for strengthening a highway network. Computers $\mathscr{E}$ Operations Research 37(10) 1708-1719. 
Pisarenko, V., M. Rodkin. 2010. Heavy-tailed distributions in disaster analysis. Springer, New York.

Rawls, C. G., M. A. Turnquist. 2010. Pre-positioning of emergency supplies for disaster response. Transportation research part B: Methodological 44(4) 521-534.

Rose, A., K. Porter, N. Dash, J. Bouabid, C. Huyck, J. Whitehead, D. Shaw, R. Eguchi, C. Taylor, T. McLane, L. Tobin, P. Ganderton, D. Godschalk, A. Kiremidjian, K. Tierney, C. West. 2007. Benefit-cost analysis of fema hazard mitigation grants. Natural Hazards Review 8(4) 97-111.

Starr, C., R. Rudman, C. Whipple. 1976. Philosophical basis for risk analysis. Annual Review of Energy 1(1) 629-662.

Stephenson, W. D., E. Bonabeau. 2007. Expecting the unexpected: The need for a networked terrorism and disaster response strategy. Homeland Security Affairs 3(1) 1-9.

Tean, E. S. 2006. Optimized positioning of pre-disaster relief force and assets. Master's thesis, Naval Postgraduate School, Monterey, CA.

Weitzman, M. L. 2011. Additive damages, fat-tailed climate dynamics, and uncertain discounting. The Economics of Climate Change: Adaptations Past and Present. University of Chicago Press, Chicago, IL, 23-46.

Yushimito, W. F., M. Jaller, S. Ukkusuri. 2012. A voronoi-based heuristic algorithm for locating distribution centers in disasters. Networks and Spatial Economics 12(1) 21-39. 